\title{
Changes in carotid blood flow after unilateral perinatal arterial ischemic stroke
}

\author{
Niek E. van der Aa', Eva D. Porsius', Jeroen Hendrikse², Britt J.M. van Kooij', Manon J.N.L. Benders', Linda S. de Vries' and \\ Floris Groenendaal'
}

INTRODUCTION: Little is known about changes in carotid blood flow after perinatal arterial ischemic stroke (PAIS). The aim of this study was to assess the blood flow in the internal carotid arteries (ICAs) after unilateral PAIS.

METHODS: The carotid flow ( $\mathrm{ml} / \mathrm{min}$ ) was measured noninvasively by means of two-dimensional phase-contrast magnetic resonance angiography (2D PC-MRA) in 25 full-term infants who had unilateral PAIS within $10 \mathrm{~d}$ after birth. In 17 infants a second 2D PC-MRA was carried out at the age of 3 mo. Asymmetry of carotid blood flow was calculated at both time points, and the circle of Willis (CoW) was assessed with a threedimensional (3D) time-of-flight MRA.

RESULTS: On the early magnetic resonance imaging (MRI), a significant increase in ipsilateral blood flow was observed (7.7\%, 95\% confidence interval (CI) 3.0-14.9\%), which persisted after correcting for CoW configuration. At 3 mo, this asymmetry was no longer observed. No relationship was found between the asymmetry in blood flow and either stroke size or postnatal age at scan.

DISCUSSION: A higher blood flow in the ipsilateral ICA was observed during the acute phase after unilateral PAIS, and this disappeared after 3 mo. Further research into the role of hyperperfusion after PAIS may suggest new approaches to neuroprotection.

t has become possible to diagnose perinatal arterial ischemic stroke (PAIS) more easily since the introduction of magnetic resonance imaging $(\mathrm{MRI})$ into neonatal care $(1,2)$. Little is known about the changes in carotid blood flow after PAIS. With the introduction of two-dimensional phase-contrast magnetic resonance angiography (2D PC-MRA), the measurement of blood flow in the carotid arteries is possible in adults $(3,4)$ as well as in neonates $(5-7)$. With the use of this technique, quantitative blood flow can be measured noninvasively.

The main arteries that feed the brain are the internal carotid arteries (ICAs) and the basilar artery (BA), which are connected at the level of the circle of Willis (CoW). Normally, cerebral blood flow (CBF) is kept relatively constant by myogenic and metabolic mechanisms and the autonomous nervous system. The latter is controlled by baroreceptors and chemoreceptors located at the carotid arteries and the aortic arch, from which signals are sent to the rostral ventrolateral medulla. Besides these control mechanisms, hypoxia can also lead to depolarization of presympathetic neurons of the rostral ventrolateral medulla, causing a sympathetically mediated rise in systemic blood pressure (8).

The relationship between PAIS and blood flow in the ICAs may improve our understanding of how PAIS develops and of the recovery and compensation mechanisms after PAIS.

In adults it has been shown that, after a stroke, the restoration of blood flow in the acute phase by means of recombinant tissue plasminogen activator restores blood flow in the viable penumbra (9). However, too high CBF may result in secondary damage through the formation of oxygen free radicals. In the latter situation, the administration of oxygen free radical scavengers may be appropriate (10). Therefore additional knowledge regarding the blood flow changes may be of importance for developing future neuroprotective strategies in infants with PAIS.

In this observational study we examined whether a relationship exists between carotid blood flow and unilateral PAIS in full-term neonates. Given that certain specific CoW configurations may increase carotid blood flow, we used a group of healthy controls to determine changes in carotid blood flow attributable to various $\mathrm{CoW}$ configurations. These data were subsequently used to correct the observed asymmetries in blood flow in the patients. Finally, we compared carotid blood flow during the first $10 \mathrm{~d}$ after the insult (early MRI) with that at a more stable phase, i.e., 3 mo after the insult (late MRI) to study potential changes in carotid blood flow.

\section{RESULTS}

Table 1 shows the patient characteristics specified for each type of infarction. Ischemia was seen in the territory of the main branch of the middle cerebral artery (MCA), other MCA branches (four in the posterior trunk of the MCA, two in the lenticulostriate branches, and eight in the cortical branches), the posterior cerebral artery (PCA), and unilateral watershed areas. Patients with ischemia of one of the smaller MCA branches formed the largest subgroup ( $n=14,56 \%)$. 
Table 1. Clinical characteristics of the studied infants, depicted as median (range)

\begin{tabular}{lcccc}
\hline & \multicolumn{4}{c}{ Involved cerebral artery } \\
\cline { 2 - 5 } Characteristic & MCA & MCA branch & PCA & Unilateral WS \\
\hline $\begin{array}{l}\text { Gender } \\
\text { (male/female) }\end{array}$ & $3 / 1$ & $7 / 7$ & $4 / 0$ & $3 / 0$ \\
$\begin{array}{l}\text { Age at first } \\
\text { MRI (d) }\end{array}$ & $4.8(0.8-5.5)$ & $4.5(1.7-10.1)$ & $4.5(4.5-5.1)$ & $4.7(4.6-6.2)$ \\
Age at second & $93(90-112)$ & $95(89-110)$ & $92(89-97)$ & $107(99-114)$ \\
MRI (d) & & & & \\
Griffiths'DQ & $98(89-112)$ & $105(90-115)$ & $98(89-100)$ & $108(102-113)$
\end{tabular}

$D Q$, developmental quotient; MCA, middle cerebral artery; MRI, magnetic resonance imaging; PCA, posterior cerebral artery; WS, watershed infarctions.

Follow-up data beyond 12 mo were available for 20 patients $(80 \%)$, none of whom were found to have a Griffiths score $<85$. Three of the infants had hemiplegia when examined at later follow-up.

\section{Reference Values of Blood Flow Asymmetry}

Analysis of the CoWs of 72 preterm infants scanned at termequivalent ages revealed a dominant A1 segment in 25 of the infants. In the posterior circulation, a unilateral or bilateral fetal PCA was observed in 42 of the infants. Nineteen infants showed no variant in the anterior or posterior circulation (adult type).

Infants with a dominant A1 segment had an increased ipsilateral blood flow, resulting in a mean asymmetry index of $11.8 \%$ $(P=<0.001$, SD $10.9 \%$, range -14.3 to $35.2 \%)$. No increase in asymmetry was observed in the group of infants with either an adult CoW or a fetal type PCA (mean asymmetry index $-3.4 \%$, SD $11.1 \%$, range -28.9 to $25.3 \%$ ).

\section{Carotid Blood Flow and Relationship With CoW}

In 22 of the 25 infants in whom early scanning was carried out three-dimensional (3D) time-of-flight (TOF) MRA images were obtained. Of these 22 infants, 10 (45.5\%) had an adulttype CoW, $6(27.3 \%$ ) had a fetal type (3 ipsilateral to the stroke and 3 contralateral to the stroke), 5 infants (22.7\%) showed hypoplasia of the A1 segment ( 3 ipsilateral to the stroke and 2 contralateral to the stroke), and 1 infant (4.5\%) had a fetal type in combination with A1 hypoplasia, both being ipsilateral to the stroke.

In 11 of the infants a second 3D-TOF was acquired at 3 mo. Two of these infants showed a change in anatomical configuration of the CoW on the TOF MRA images, with ipsilateral hypoplastic A1 segments on the early MRI and no ipsilateral hypoplasia on the late MRI. In one infant an ipsilateral fetal type configuration that was seen on the early MRI was not present on the late MRI.

An increase in ipsilateral carotid blood flow was observed on the early scan (mean asymmetry index 7.7\%, 95\% confidence interval (CI) $1.5-13.8 \%, P=0.02$ ). This is illustrated in Figure 1a, in which the asymmetry of the carotid blood flow is plotted against the postnatal age on the early MRI, categorized by type of CoW. An analysis of the different
CoW subgroups showed that only infants with an adult CoW on the early MRI $(n=10)$ showed a significant asymmetry of carotid blood flow (mean 10.7\%, $P=0.01$, 95\% CI $4.8-16.5 \%)$.

For the 22 infants with a known CoW on the early MRI, the asymmetry indexes (converted to a $Z$ value) are depicted in Figure 1b. Again, an increase in asymmetry was observed, depicted by a mean $Z$ value of 1.05 ( $P=0.001,95 \%$ CI 0.50 1.61). Subanalysis of the different types of CoW configurations showed an increased $Z$ value not only in the adult CoW group (mean $Z$ value $1.15, P=0.001$, 95\% CI 0.66-1.64) but also in the A1 hypoplasia group (mean $\mathrm{Z}$ value $1.51, P=0.02,95 \% \mathrm{CI}$ $0.37-2.66)$.

\section{Carotid Blood Flow and Time Elapsed After the Infarction}

No significant correlation was found between the age at which the early scan was carried out and the asymmetry in carotid blood flow. A total of 17 infants were scanned again at the age of 3 mo. The mean asymmetry of carotid flow in this subgroup decreased from $8.5 \%$ on the early MRI to $-1.0 \%$ on the late MRI $(P=0.03)$. The asymmetry of blood flow at 3 mo was no longer significantly different from zero $(-1.0 \%, 95 \%$ CI -4.3 to $2.3 \%)$.

Conversion of the asymmetry index to a $Z$ value for the 11 infants whose CoW configurations were known at 3 mo of age also resulted in no significant difference in carotid blood flow (Figure 2, mean $Z$ value $0.07,95 \% \mathrm{CI}-0.5$ to 0.7 ). However, an analysis of the $Z$ values on the early MRI for these 11 infants still showed a significant asymmetry (mean $Z$ value $1.18, P=$ 0.02, 95\% CI 0.41-2.0).

\section{Carotid Blood Flow and Type and Size of Lesion}

The carotid blood flow was significantly higher on the affected side in the MCA subgroup (both main and smaller branches, mean asymmetry index $8.7 \%(P=0.02,95 \%$ CI $1.7-15.6 \%$, Figure 3)) but not in the PCA and unilateral watershed subgroups. On the late MRI no significant asymmetry was found in any of the subtypes of lesions.

The mean stroke volume was found to be $21.0 \mathrm{ml}$ (range $0.09-112 \mathrm{ml}$ ). The mean lesion size, expressed as the percentage of the affected hemisphere that was ischemic, was $11.2 \%$ (range 0.06-62.1\%). No correlation was found between the lesion size and the asymmetry index or $Z$ value.

\section{DISCUSSION}

In this study with infants who suffered unilateral PAIS, the blood flow in the ICA ipsilateral to ischemic brain tissue was significantly higher than in the contralateral ICA in the first $10 \mathrm{~d}$ of life. This suggests rapid restoration of blood flow in the distal cerebral artery and subsequent hyperperfusion of its territory. Subsequently we demonstrated that this asymmetry no longer persists at 3 mo after the insult.

\section{Carotid Blood Flow and Time After the Infarction}

Postischemichyperperfusion is a phenomenon that is frequently observed in adults and in animal models; it is also known as 

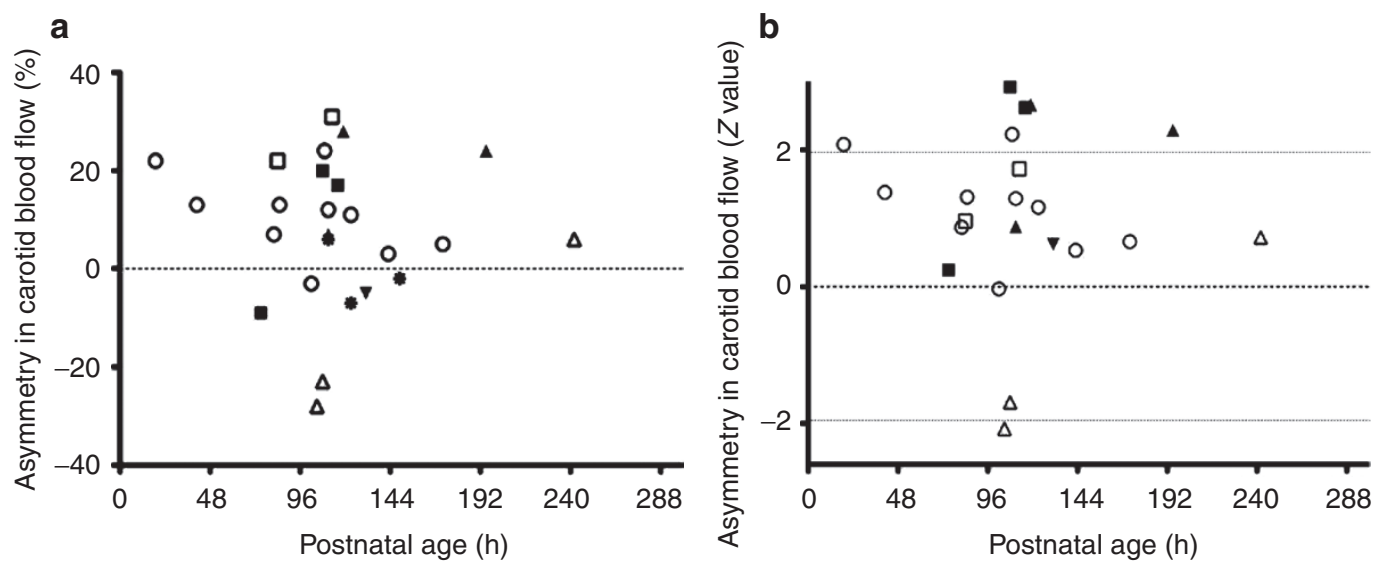

Figure 1. The asymmetry of carotid blood flow as observed at the early MRI depicted for the different CoW configurations. (a) An increase in ipsilateral blood flow, expressed by the asymmetry index, can be observed. (b) This increase can still be observed after correcting for the type of CoW. The broken lines at $Z$ $=-1.96$ and $Z=1.96$ correspond with $95 \%$ of the observed asymmetries in the control group, after correcting for the type of CoW. Adult type (open circles); ipsilateral fetal type (shaded triangles); contralateral fetal type (open triangles); hypoplasia ipsilateral A1 (shaded squares); hypoplasia contralateral A1 (open squares); hypoplasia ipsilateral A1 and fetal type (shaded inverted triangles); unknown (asterisks). CoW, circle of Willis; MRI, magnetic resonance imaging.

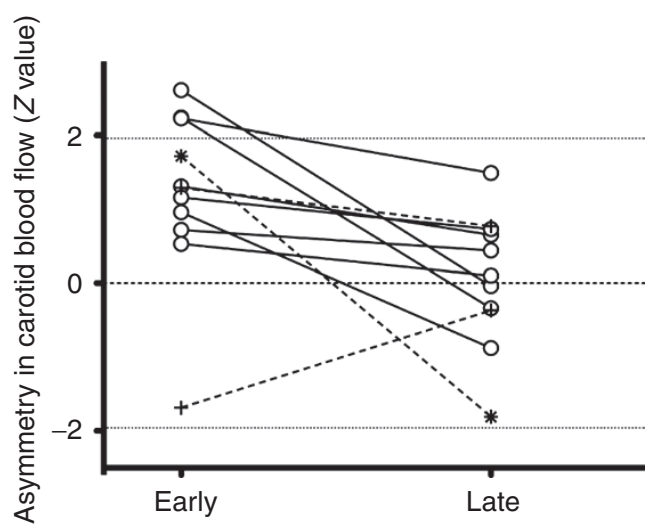

Figure 2. The course of asymmetry in blood flow in the ICAs over time. Each line connects the asymmetry at the early MRI with the data on the late MRI for each individual patient for each affected artery. The asymmetry that can be observed on early MRI can no longer be observed on late MRI. The broken lines at $Z=-1.96$ and $Z=1.96$ correspond with $95 \%$ of the observed asymmetries in the control group, after correcting for the type of CoW. MCA branches (open circles), PCA (crosses), and unilateral watershed infarctions (asterisks). CoW, circle of Willis; ICA, internal carotid artery; MCA, middle cerebral artery; MRI, magnetic resonance imaging; $P C A$, posterior cerebral artery.

"luxury perfusion" or "reactive hyperemia" when the blood flow exceeds the metabolic needs of the brain (11-13). The underlying mechanism is a rapid increase in anerobic metabolism after hypoxia-ischemia. The resulting by-products, including lactate and adenosine, can induce vasodilation through relaxation of the vascular smooth muscle, thereby decreasing the vascular resistance. In addition to the effects of vasoactive metabolites, other mechanisms, including neurogenic vasodilation, have been proposed (14).

Previous studies in infants focused mainly on changes in CBF velocity (CBFV) measured using Doppler ultrasonography in infants with neonatal encephalopathy (NE) (15-17). Higher mean CBFV values have been found during the first days after asphyxia in infants with severe NE as compared with a control group of infants with mild to moderate NE and healthy infants, the maximum values of CBFV being seen at $36-72 \mathrm{~h}$ after birth $(16,17)$. More recently, using perfusionweighted imaging, Wintermark et al. reported observing initial hypoperfusion followed by hyperperfusion on days 2 and 3 in infants who develop brain injury after NE (18). In contrast, no subsequent hyperperfusion was observed in the infants who did not go on to develop brain injury. Changes in CBFV after PAIS have also been reported in several smaller studies using Doppler ultrasound or xenon clearance (19-24). Both increases and decreases in ipsilateral CBFV have been reported. Perlman et al. reported that a transient decrease in ipsilateral CBFV was seen in four of eight infants studied, and that this disappeared within $24 \mathrm{~h}$ after the initial investigation, thereby suggesting that the timing of the investigation plays an important role (24).

On the basis of clinical and MRI findings, it is likely that PAIS occurs within a very limited time frame around the time of birth $(25,26)$. In our cohort, the cause of the PAIS often remained unknown. None of the patients had carotid or cardiac abnormalities as ascertained by ultrasonography, nor was there any abnormal anatomy of blood vessels seen on MRA. These findings suggest that the stroke was postnatal. In animal models of stroke, hyperperfusion has been observed but is not constant during the first few days after ischemia $(27,28)$. In our clinical study, only a single measurement was performed during the first week. This precluded the opportunity to observe any changes in (hyper)perfusion. Serial measurements would allow for such observations to be recorded and might be of value for predicting tissue viability, given that both hyper- and hypoperfusion have been associated with infarction $(27,29)$. Knowledge regarding the relationship between perfusion and (tissue viability) outcome may therefore open up potential new approaches to neuroprotection.

\section{Carotid Blood Flow and Type and Size of Lesion}

No relationship was found between blood flow asymmetry and the size of the lesion. However, infants with a stroke in the MCA or one of its branches tended to have a higher ipsilateral 

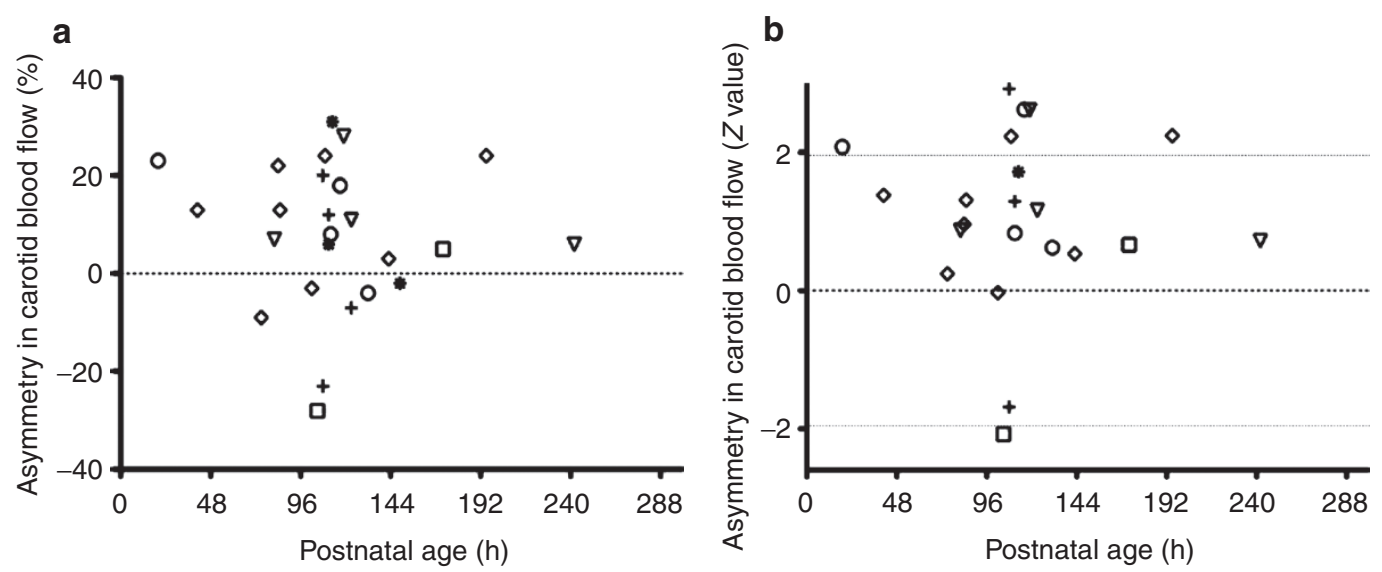

Figure 3. The asymmetry of carotid blood flow as observed at the early MRI, depicted for the different arterial branches involved. (a) Asymmetry of carotid blood flow and (b) asymmetry expressed as a $Z$ value after correcting for the type of CoW vs. age at scan, depicted for the different strokes observed. Main branch MCA (open circles); posterior MCA branch (open inverted triangles); lenticulostriate MCA branch (open squares); cortical MCA branch (open diamonds); PCA (crosses); unilateral watershed infarctions (asterisks). The broken lines at $Z=-1.96$ and $Z=1.96$ correspond with $95 \%$ of the observed asymmetries in the control group, after correcting for the type of CoW. CoW, circle of Willis; MCA, middle cerebral artery; PCA, posterior cerebral artery.

carotid blood flow and a reduction in asymmetry 3 mo later. This was not found in the other two subgroups. For the PCA strokes the absence of an asymmetry of carotid blood flow might be explained by the fact that the vertebral arteries and subsequently the BA, rather than the ICAs, supply the posterior regions of the brain.

\section{Carotid Blood Flow and Relationship With CoW}

It has been shown that carotid blood flow is influenced by the type of CoW. Tanaka et al. reported that the relative contributions of the ICAs and the BA to carotid flow correlate significantly with the variations in the anatomy of the CoW in healthy adults (3). van Kooij et al. have reported that this is also true for preterm infants examined at term-equivalent ages (5).

The respective ratios of estimated distribution of blood flow to the ICAs and the BA are 40:40:20, 30:50:20, and 50:40:10 in the adult type, A1 hypoplasia type, and P1 hypoplasia (or fetal) type, respectively (3). van Kooij et al. found that there was no asymmetry in blood flow in the ICAs of infants with an adult or fetal type anatomy of the CoW, and that there was a decrease in flow in the ICA ipsilateral to a hypoplastic or absent A1 segment as compared with the flow in the contralateral ICA (5).

In our study we took the different types of CoW into account while interpreting the asymmetries in carotid blood flow. This was of special importance with respect to the infants with a hypoplastic A1 segment. Any increase in ipsilateral blood flow may be obscured in the presence of an ipsilateral hypoplastic A1 segment, whereas a contralateral A1 hypoplasia is likely to result in an increased ipsilateral blood flow, which does not necessarily reflect an increase in blood flow because of the stroke. The absence in some cases of any increase in flow, as determined with the asymmetry index, and the presence of increased flow after converting the indexes to $Z$ values, underlines the importance of this correction.

Early hyperperfusion has been reported to be both beneficial (by reducing the infarct extension and minimizing neuronal damage in the penumbra) as well as harmful (by aggravating edema, inducing secondary hemorrhages, producing damagecausing free radicals and cytokines, and inducing influx and activation of inflammatory cells (30-32)). In adults, a better understanding of the interruption in $\mathrm{CBF}$ after ischemic stroke led to the use of tissue plasminogen activator; to date this is the only available therapy for the acute phase (33). No therapies are available for neonates with PAIS, but a better understanding of the role of perfusion after the insult might lead to new insights and targets for therapeutic intervention like the administration of oxygen free radical scavengers (10). The usage of perfusionweighted imaging combined with diffusion-weighted imaging (DWI) might help to identify regions of hyperperfusion and salvageable penumbra.

Currently, no reference data for blood flow in the ICAs of healthy, full-term neonates have been published. Only two studies have reported PC-MRA-measured CBF values in neonates; however, neither of these studies was carried out in healthy, full-term infants $(5,7)$. Furthermore, these studies did not report the blood flow in relation to total brain volume. In the Netherlands, ethical considerations preclude MRI examination of healthy neonates, thereby limiting the available reference data to those relating to preterm infants. The availability of reference values in healthy, full-term infants, adjusted for total brain volume, would allow a more reliable quantification of changes in carotid blood flow.

The majority of the infants in our study had either clinical or subclinical seizures, and therefore received phenobarbital. In animal models, phenobarbital has been shown to reduce CBF, whereas studies in neonates have shown little to no effect of this drug on $\mathrm{CBF}(34,35)$. It is unlikely that phenobarbital could have affected the symmetry of CBF in our study patients. However, the presence of phenobarbital and other sedatives or anticonvulsants could limit comparisons of the flow values with those in healthy controls.

A limitation of this study is the relatively small number of infants studied. Asymmetry of CBF probably depends on multiple factors; apart from the type of CoW, the extent of ischemia 
and the presence and timing of recanalization may play roles. Despite this multifactorial dependency, we were able to show an increased ipsilateral blood flow during the acute phase. Larger studies will be needed to disentangle the roles of these different factors.

Another limitation was that it was not possible to record longitudinal measurements during the first week after the stroke. Little is known about the changes in carotid blood flow within individual infants during the first hours and days after birth. The intensive care setting in which these infants were placed precluded repetitive measurements during the first week. In the future, the use of dedicated MR incubators may facilitate early repeat MRIs. Finally, patients were scanned on either a $1.5 \mathrm{~T}$ system or a $3 \mathrm{~T}$ system. However, given that we used asymmetry indexes rather than absolute values for making comparisons, the difference in scanners is unlikely to have influenced our results.

\section{Conclusions}

In infants with unilateral PAIS, blood flow in the ipsilateral ICA was significantly higher than in the contralateral ICA between days 2 and 10. This finding was independent of the CoW configuration. On the MRI carried out after $3 \mathrm{mo}$, the asymmetry in carotid blood flow had disappeared. The importance of this higher ipsilateral blood flow in promoting recovery from stroke or, by contrast, in causing additional injury by releasing oxygen free radicals, remains to be established.

\section{METHODS}

\section{Subjects}

Between the years 2006 and 2010, a total of 36 full-term infants with unilateral PAIS (as detected by MRI) were admitted to our neonatal intensive care unit. Judging from clinical and MRI findings, these incidents of stroke were likely to have occurred around the time of birth.

The infants included in this study were those in whom 2D PC-MRA was acquired within $10 \mathrm{~d}$ of birth, and in whom DWI revealed unilateral ischemia. These criteria were met for 25 of the infants. MRI was repeated at the age of 3 mo in all the infants, but only 17 infants had a second PC-MRA. The ethics committee of the University Medical Center Utrecht waived the requirement to obtain informed consent for this study.

\section{MRI Protocol}

The infants were placed in a vacuum fixation pillow (Med-Tec, Orange City, IA) during the MRI examination. They were sedated with an intramuscular injection containing a mixture of $2 \mathrm{mg} / \mathrm{kg}$ pethidine, $0.5 \mathrm{mg} / \mathrm{kg}$ chlorpromazine, and $0.5 \mathrm{mg} / \mathrm{kg}$ promethazine, as reported previously (36). Monitoring was performed using pulse oximetry (Nonin, Minneapolis, MN), and respiratory rate was recorded using standard Philips equipment (Philips Medical Systems, Best, The Netherlands). For hearing protection, Minimuffs (Natus Medical, San Carlos, CA) were used. During the whole examination, a neonatologist was present to ensure patient safety.

The infants were scanned using either a $1.5 \mathrm{~T}$ or a $3.0 \mathrm{~T}$ whole-body MR system (Achieva platform; Philips Medical Systems) depending on availability. A T2-weighted image, DWI, 2D PC-MRA, and (when time allowed) a 3D TOF-MRA, were acquired in the first $10 \mathrm{~d}$ of life (early MRI) and often also at $\sim 3$ mo after the insult (late MRI).

To measure blood flow in the ICAs and in the BA, a 2D PC section was positioned perpendicular to the BA, just blow the carotid siphon, determined on the basis of a localizer MRA slab in the sagittal plane (Figure 4; 1.5T protocol: repetition time (TR) $13-16 \mathrm{~ms}$, echo time (TE) $8-10 \mathrm{~ms}$, flip angle $7.5^{\circ}$, section thickness $5 \mathrm{~mm}$, field of
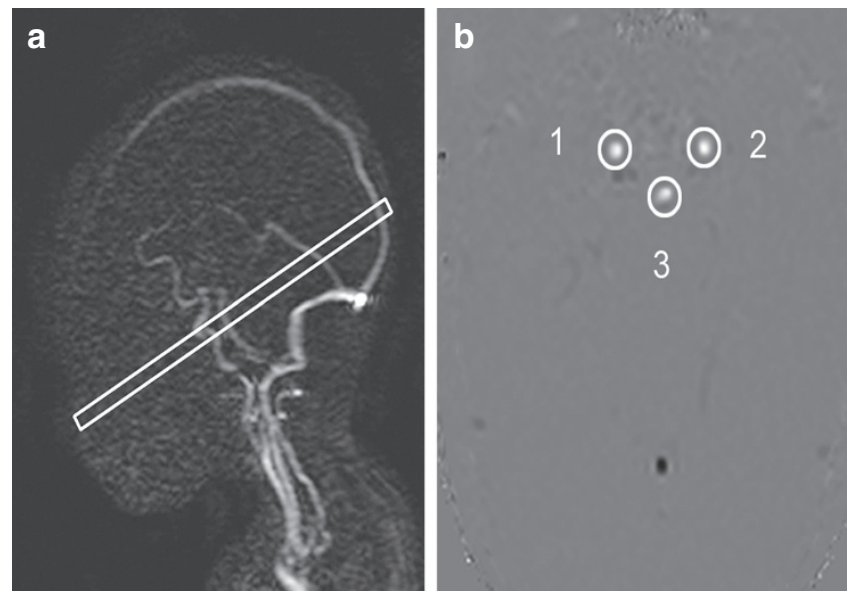

Figure 4. MRA measurement of carotid blood flow. (a) Sagittal localizer MRA slab with the 2D PC section positioned perpendicular to the BA, just below the carotid siphon. (b) Axial view of 2D PC section with measurements of (1) the right and (2) the left ICA, and (3) the BA. 2D, two-dimensional; BA, basilar artery; ICA internal carotid artery; MRA, magnetic resonance angiography; $\mathrm{PC}$, phase contrast.

view $150 \times 103 \mathrm{~mm}$, matrix, $256 \times 256,8$ averages, velocity sensitivity $30 \mathrm{~cm} / \mathrm{s}$; the $3 \mathrm{~T}$ protocol was identical except for a flip angle of 10 ). Quantitative flow values $(\mathrm{ml} / \mathrm{min}$ ) were calculated in the ICAs and the BA separately by integrating across manually drawn regions of interest enclosing the lumen of the relevant vessel, as reported previously (6). Measurements were performed twice by the same examiner (E.D.P.), with an intra-observation interval of 3 mo. Asymmetry in blood flow between the ICAs was calculated as $100 \% \times$ (flow in the ipsilateral ICA - flow in the contralateral ICA)/flow in both ICAs combined.

The 3D TOF-MRA with a subsequent maximum-intensity projection reconstruction was acquired to assess the anatomy of the CoW (1.5T protocol: TR $25 \mathrm{~ms}$, TE $6.9 \mathrm{~ms}$, flip angle $20^{\circ}$, field of view $200 \times$ $200 \mathrm{~mm}$, matrix size $512 \times 512,2$ averages, section thickness $0.5 \mathrm{~mm}$, 140 sections; 3T protocol: TR $23 \mathrm{~ms}$, TE $3.5 \mathrm{~ms}$, flip angle $18^{\circ}$, field of view $160 \times 128 \mathrm{~mm}$, matrix size $512 \times 512$, 2 averages, section thickness $1 \mathrm{~mm}$ with $0.5 \mathrm{~mm}$ overlap, 60 sections).

The TOF-MRAs were scored by an experienced examiner (J.H., ref. (4-6)) in accordance with the classification of the anatomy of the CoW as described by Krabbe-Hartkamp et al. $(5,37)$. In this classification, the anatomy of the CoW was divided into: (i) a normal adult type, (ii) a fetal-type PCA, and (iii) hypoplasia of the A1 segment. The fetal-type and adult-type CoWs are illustrated in Figure 5.

\section{Reference Values for Blood Flow Asymmetry}

Blood flow measurements of a previously published cohort of 72 infants born preterm and scanned at term-equivalent age, were used to create reference values of the asymmetry of carotid blood flow (5). All these infants were scanned using a 2D-PC MRA and a 3D-TOF MRA on a 3.0T MR system, as described earlier in this article. Asymmetry indexes of carotid blood flow in these reference infants were calculated in the same manner as for the study infants with PAIS.

van Kooij et al. have previously demonstrated that carotid blood flow increases in the presence of an ipsilateral dominant A1 segment (i.e., contralateral A1 hypoplasia), whereas there was no asymmetry in carotid blood flow in infants with an adult-type CoW or a fetal-type PCA. If an asymmetry of carotid blood flow is observed after a PAIS, it is necessary to take into account the type of CoW of the patient; this is because a dominant A1 segment on the side of the ischemic lesion may increase the asymmetry of flow, whereas a contralateral dominant A1 segment may diminish the asymmetry. We therefore performed a subanalysis in infants whose CoW configuration was known, correcting the asymmetry data after adjusting for the CoW configuration. This was done by converting the blood flow asymmetry in each patient to a $Z$ value ((asymmetry in patient - mean 


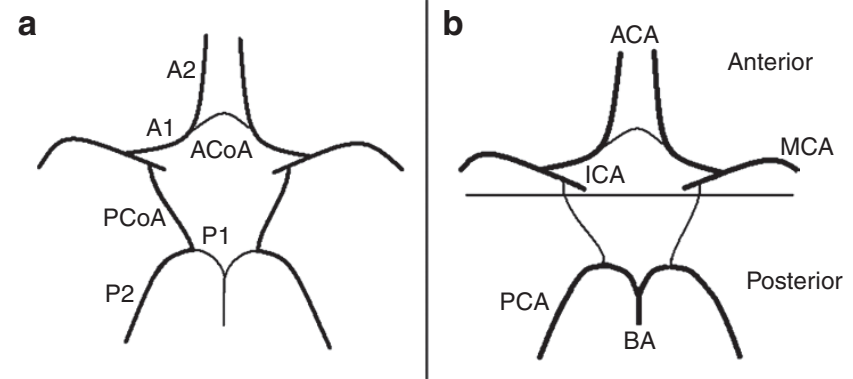

Figure 5. Variations in the anatomy of the circle of Willis. (a) The fetal-type CoW, in which all segments have an identical size and the internal carotid artery (ICA) provides blood to all ipsilateral cerebral arteries. (b) The adult-type CoW, in which the ICA provides blood to the ipsilateral anterior cerebral artery (ACA) and middle cerebral artery (MCA), while the basilar artery (BA) supplies blood to both posterior cerebral arteries (PCAs). Reproduced with permission from the Radiological Society of North America, from Figures 1 and 2 in ref. 37. ACoA, anterior communicating artery; CoW, circle of Willis; $\mathrm{PCoA}$, posterior communicating artery.

asymmetry in controls)/SD of asymmetry in controls). This was done separately for the infants with an adult-type CoW or fetal-type PCA and for those with a dominant A1 segment.

\section{Analysis of Lesion Size}

For DWI, a single-shot echo-planar imaging was used in three nonorthogonal directions, using a $b$ value of $1,000 \mathrm{~s} / \mathrm{mm}^{2}(1.5 \mathrm{~T})$ or $800 \mathrm{~s} /$ $\mathrm{mm}^{2}(3.0 \mathrm{~T})$ and one $b=0 \mathrm{~s} / \mathrm{mm}^{2}$ image $(25$ axial slices of $4 \mathrm{~mm} ; 1.5 \mathrm{~T}$ protocol: TR 4,000 ms, TE $89 \mathrm{~ms}$, field of view $180 \times 180 \mathrm{~mm}$, matrix size $256 \times 256$; 3.0 T protocol: TR 2,400 ms, TE $68 \mathrm{~ms}$, field of view 230 $\times 230 \mathrm{~mm}$, matrix size $256 \times 256$ ).

The lesion size was approximated by manually delineating the stroke volume on the apparent diffusion coefficient map derived from the DWI. This volume was expressed as a percentage of hemispheric volume, as delineated on the DWI, which included the affected hemisphere above the superior cerebral peduncle and excluded the cerebellum.

\section{Follow-Up}

Neurodevelopmental outcome was evaluated at regular intervals after the discharge of the patient as part of our follow-up program. Neurodevelopment was assessed using the Griffiths' mental development scale at the ages of 3, 9, and 18 mo (38). Postural and motor control were assessed by a pediatric physiotherapist who was unaware of the clinical history and imaging findings.

\section{Data Analyses}

SPSS version 15.0 (SPSS, Chicago, IL) was used for the data analyses. The relationship between the asymmetry of carotid blood flow measured during early MRI and (i) the postnatal age at MRI and (ii) lesion size were analyzed using the Pearson correlation coefficient. The one-sample $t$-test or the one-sample Wilcoxon signed-rank test was used to determine whether the asymmetry was significantly different from zero. For all tests a value of $P<0.05$ was considered statistically significant. Whenever possible, $95 \%$ CIs were calculated.

\section{ACKNOWLEDGMENTS}

The authors thank the MRI technicians of University Medical Center Utrecht for their help in carrying out the examinations of the patients.

\section{STATEMENT OF FINANCIAL SUPPORT}

N.E.v.d.A. was supported by grants from the Wilhelmina Children's Hospital Research Fund and the Dutch Phelps Foundation (http://www.phelpsstichting.nl).

\section{REFERENCES}

1. Lequin MH, Dudink J, Tong KA, Obenaus A. Magnetic resonance imaging in neonatal stroke. Semin Fetal Neonatal Med 2009;14:299-310.
2. Benders MJ, Groenendaal F, De Vries LS. Preterm arterial ischemic stroke. Semin Fetal Neonatal Med 2009;14:272-7.

3. Tanaka H, Fujita N, Enoki T, et al. Relationship between variations in the circle of Willis and flow rates in internal carotid and basilar arteries determined by means of magnetic resonance imaging with semiautomated lumen segmentation: reference data from 125 healthy volunteers. AJNR Am J Neuroradiol 2006;27:1770-5.

4. Hendrikse J, van Raamt AF, van der Graaf Y, Mali WP, van der Grond J. Distribution of cerebral blood flow in the circle of Willis. Radiology 2005;235:184-9.

5. van Kooij BJ, Hendrikse J, Benders MJ, de Vries LS, Groenendaal F. Anatomy of the circle of Willis and blood flow in the brain-feeding vasculature in prematurely born infants. Neonatology 2010;97:235-41.

6. Hendrikse J, de Vries LS, Groenendaal F. Magnetic resonance angiography of cerebral arteries after neonatal venoarterial and venovenous extracorporeal membrane oxygenation. Stroke 2006;37:e15-7.

7. Benders MJ, Hendrikse J, De Vries LS, Van Bel F, Groenendaal F. Phasecontrast magnetic resonance angiography measurements of global cerebral blood flow in the neonate. Pediatr Res 2011;69:544-7.

8. Guyenet PG. Neural structures that mediate sympathoexcitation during hypoxia. Respir Physiol 2000;121:147-62.

9. Paciaroni M, Caso V, Agnelli G. The concept of ischemic penumbra in acute stroke and therapeutic opportunities. Eur Neurol 2009;61:321-30.

10. Buonocore G, Groenendaal F. Anti-oxidant strategies. Semin Fetal Neonatal Med 2007;12:287-95.

11. Aries MJ, Elting JW, De Keyser J, Kremer BP, Vroomen PC. Cerebral autoregulation in stroke: a review of transcranial Doppler studies. Stroke 2010;41:2697-704.

12. Soin JS, Burdine JA. Acute cerebral vascular accident associated with hyperperfusion. Radiology 1976;118:109-12.

13. Lassen NA. The luxury-perfusion syndrome and its possible relation to acute metabolic acidosis localised within the brain. Lancet 1966;2:1113-5.

14. Macfarlane R, Moskowitz MA, Sakas DE, Tasdemiroglu E, Wei EP, Kontos HA. The role of neuroeffector mechanisms in cerebral hyperperfusion syndromes. J Neurosurg 1991;75:845-55.

15. Bennhagen RG, Weintraub RG, Lundström NR, Svenningsen NW. Hypoxic-ischaemic encephalopathy is associated with regional changes in cerebral blood flow velocity and alterations in cardiovascular function. Biol Neonate 1998;73:275-86.

16. Ilves P, Lintrop M, Talvik I, Muug K, Maipuu L. Changes in cerebral and visceral blood flow velocities in asphyxiated term neonates with hypoxicischemic encephalopathy. J Ultrasound Med 2009;28:1471-80.

17. Ilves P, Lintrop M, Talvik I, Muug K, Maipuu L, Metsvaht T. Low cerebral blood flow velocity and head circumference in infants with severe hypoxic ischemic encephalopathy and poor outcome. Acta Paediatr 2009;98:459-65.

18. Wintermark P, Hansen A, Gregas MC, et al. Brain perfusion in asphyxiated newborns treated with therapeutic hypothermia. AJNR Am J Neuroradiol 2011;32:2023-9.

19. Messer J, Haddad J, Casanova R. Transcranial Doppler evaluation of cerebral infarction in the neonate. Neuropediatrics 1991;22:147-51.

20. Nir A, Perles Z, Gomori JM, Rein AJ. Echocardiographic sign for cerebral ischemia. Pediatr Neurol 1997;17:259-61.

21. Nishimaki S, Seki K, Yokota S. Cerebral blood flow velocity in two patients with neonatal cerebral infarction. Pediatr Neurol 2001;24:320-3.

22. Steventon DM, John PR. Power Doppler ultrasound appearances of neonatal ischaemic brain injury. Pediatr Radiol 1997;27:147-9.

23. Pryds O, Greisen G, Lou H, Friis-Hansen B. Vasoparalysis associated with brain damage in asphyxiated term infants. J Pediatr 1990;117(1 Pt 1):119-25.

24. Perlman JM, Rollins NK, Evans D. Neonatal stroke: clinical characteristics and cerebral blood flow velocity measurements. Pediatr Neurol 1994;11:281-4.

25. Cowan F, Rutherford M, Groenendaal F, et al. Origin and timing of brain lesions in term infants with neonatal encephalopathy. Lancet 2003;361:736-42.

26. Dudink J, Mercuri E, Al-Nakib L,et al. Evolution of unilateral perinatal arterial ischemic stroke on conventional and diffusion-weighted MR imaging. AJNR Am J Neuroradiol 2009;30:998-1004. 


\section{Articles $\mid$ vander Aa et al.}

27. Shen Q, Du F, Huang S, Duong TQ. Spatiotemporal characteristics of postischemic hyperperfusion with respect to changes in T1, T2, diffusion, angiography, and blood-brain barrier permeability. J Cereb Blood Flow Metab 2011;31:2076-85.

28. Heiss WD, Graf R, Löttgen J, et al. Repeat positron emission tomographic studies in transient middle cerebral artery occlusion in cats: residual perfusion and efficacy of postischemic reperfusion. J Cereb Blood Flow Metab 1997; 17:388-400.

29. Viallon M, Altrichter S, Pereira VM, et al. Combined use of pulsed arterial spin-labeling and susceptibility-weighted imaging in stroke at 3T. Eur Neurol 2010;64:286-96.

30. Kato H, Kogure K. Biochemical and molecular characteristics of the brain with developing cerebral infarction. Cell Mol Neurobiol 1999;19:93-108.

31. Sugawara T, Chan PH. Reactive oxygen radicals and pathogenesis of neuronal death after cerebral ischemia. Antioxid Redox Signal 2003;5:597-607.

32. Perlman JM. Intervention strategies for neonatal hypoxic-ischemic cerebral injury. Clin Ther 2006;28:1353-65.
33. Tissue plasminogen activator for acute ischemic stroke. The National Institute of Neurological Disorders and Stroke rt-PA Stroke Study Group. N Engl J Med 1995;333:1581-87.

34. Scalais E, Beharry K, Papageorgiou A, Bureau M, Aranda JV. Effects of phenobarbital on cerebral blood flow in the newborn piglet. Dev Pharmacol Ther 1992;19:10-8.

35. Andersen K, Jensen KA, Ebbesen F. The effect of phenobarbital on cerebral blood flow in newborn infants with foetal distress. Eur J Pediatr 1994;153:584-7.

36. Groenendaal F, Veenhoven RH, van der Grond J, Jansen GH, Witkamp TD, de Vries LS. Cerebral lactate and N-acetyl-aspartate/choline ratios in asphyxiated full-term neonates demonstrated in vivo using proton magnetic resonance spectroscopy. Pediatr Res 1994;35:148-51.

37. Krabbe-Hartkamp MJ, van der Grond J, de Leeuw FE, et al. Circle of Willis: morphologic variation on three-dimensional time-of-flight MR angiograms. Radiology 1998;207:103-11.

38. Griffiths R. The Abilities of Babies: A Study in Mental Measurement. London: University of London Press, 1976 